\title{
Neurological and psychiatric presentations associated with COVID-19
}

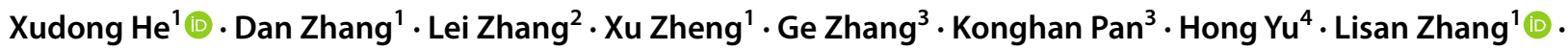 \\ Xingyue $\mathrm{Hu}^{1}$
}

Received: 2 November 2020 / Accepted: 24 February 2021 / Published online: 12 March 2021

(c) Springer-Verlag GmbH Germany, part of Springer Nature 2021

\begin{abstract}
The objective is to investigate coronavirus disease 2019 (COVID-19)-associated neurological and psychiatric effects and explore possible pathogenic mechanisms. This study included 77 patients with laboratory-confirmed COVID-19 in Wuhan, China. Neurological manifestations were evaluated by well-trained neurologists, psychologists, psychiatric presentations and biochemical changes were evaluated using the Generalized Anxiety Disorder 7-item scale, Patient Health Questionnaire-9, Brief Psychiatric Rating Scale, and electronic medical records. Eighteen (23.4\%) patients presented with neurological symptoms. Patients with neurological presentations had higher urea nitrogen, cystatin $\mathrm{C}$, and high-sensitivity $\mathrm{C}$-reactive protein levels and lower basophil counts. Among them, patients with muscle involvement had higher urea nitrogen and cystatin $\mathrm{C}$ levels but lower basophil counts. In addition, patients with psychiatric presentations were older and had higher interleukin (IL)-6 and IL-10 levels and higher alkaline phosphatase, R-glutamate transferase, and urea nitrogen levels. Moreover, patients with anxiety had higher IL-6 and IL-10 levels than those without, and patients with moderate depression had higher $\mathrm{CD} 8+\mathrm{T}$ cell counts and lower CD4 +/CD8 + ratios than other patients. This study indicates that the central nervous system may be influenced in patients with COVID-19, and the pathological mechanisms may be related to direct virus invasion of the central nervous system, infection-mediated overreaction of the immune system, and aberrant serum pro-inflammatory factors. In addition, basophils and cystatin $\mathrm{C}$ may also play important roles during these pathological processes. Our findings suggest that neurological and psychiatric presentations should be evaluated and managed in patients with COVID-19. Further studies are needed to investigate the underlying mechanisms.
\end{abstract}

Keywords COVID-19·SARS-CoV-2 $\cdot$ Neurological presentations $\cdot$ Psychiatric presentations

\section{Introduction}

In December 2019, unexplained cases of pneumonia emerged in Wuhan, China, and this acute respiratory infection rapidly spread to other regions of China, followed by outbreaks in other Asian countries, Europe, and North

Xingyue $\mathrm{Hu}$

huxingyue2003@zju.edu.cn

1 Department of Neurology, Sir Run Run Shaw Hospital, School of Medicine, Zhejiang University, Hangzhou, China

2 Department of Psychiatry, Sir Run Run Shaw Hospital, School of Medicine, Zhejiang University, Hangzhou, China

3 Department of Critical Medicine, Sir Run Run Shaw Hospital, School of Medicine, Zhejiang University, Hangzhou, China

4 Department of General Surgery, Sir Run Run Shaw Hospital, School of Medicine, Zhejiang University, Hangzhou, China
America. By March 19, 2020, there were 80,967 coronavirus disease 2019 (COVID-19) cases confirmed in China, including 3248 deaths [1]; globally 209,839 cases were diagnosed with 8778 deaths [2]. COVID-19 has been confirmed to originate from a novel coronavirus, with $96 \%$ sequence homology with that of the bat coronavirus [3]. Although the pathogen responsible for COVID-19 and the severe acute respiratory syndrome coronavirus (SARS-CoV) of 2003 results in similar symptoms and have similar pathogen gene sequences, the pathogen responsible for COVID-19 is different from SARS-CoV. Therefore, on February 11, 2020, this novel coronavirus was named SARS coronavirus 2 (SARSCoV-2) by the International Committee on Taxonomy of Viruses [4, 5].

Coronavirus was first isolated in 1937, and soon thereafter, other types of coronaviruses were discovered in different animals. The first human coronavirus was cultivated from the nasal cavities in 1965 [6]. Although most human 
coronavirus infections are mild, the epidemic of severe acute respiratory syndromes caused by SARS-CoV infection in 2003 reminds us that coronavirus can transmit across species, causing human infection with severe clinical symptoms and a high mortality rate. This was confirmed by the epidemic situation caused by the Middle East respiratory syndrome (MERS) coronavirus and SARS-CoV-2 [7-9].

Receptors of SARS-CoV include angiotensin-converting enzyme 2 (ACE2) and CD26. ACE2, a zinc-dependent peptidase that interacts with protein $\mathrm{S}$, plays an important role in mediating virus invasion into cells, promoting virus replication and aggravating acute lung injury, which has been identified as a functional receptor of SARS-CoV-2 [3]. The distribution of ACE2 is closely related to the location of virus invasion as well as clinical manifestations. Accumulating evidence has demonstrated that SARS-CoV-2 invades the mucus membrane of the respiratory and digestive tracts and results in multiple system damage, especially in the respiratory and hematological systems, presenting as severe interstitial pneumonia and progressive lymphopenia [4].

Respiratory symptoms, muscle soreness, and fatigue have been observed in COVID-19, and severe COVID-19 cases have much higher interleukin (IL)-2, IL-7, IL-10, and tumor necrosis factor (TNF)- $\alpha$ levels than non-severe cases [5]. Notably, a recent study found that patients with COVID-19 had higher levels of depression and anxiety than healthy controls [10]. However, the relationship between neurological and psychiatric presentations and expression levels of pro-inflammatory factors and serum $\mathrm{T}$ cell subsets remains unclear. In this study, we examined neurological and psychiatric presentations in patients diagnosed with COVID19 in Wuhan, China, and analyzed the correlation of these presentations with pro-inflammatory factors and serum $\mathrm{T}$ cell subsets to provide a theoretical basis for neurological involvement in COVID-19. Considering that psychiatric presentations might suggest brain damage, we also determined initial brain damage symptoms in some cases in this study.

\section{Methods}

This descriptive study investigated clinical manifestations of patients with SARS-CoV-2-infected pneumonia. We included patients with COVID-19 who were admitted to the isolation ward of the cancer center at Union Hospital, Tongji Medical College, Huazhong University of Science and Technology, Wuhan, Hubei, China between February 14 and March 14, 2020. Clinical data were systematically collected and investigated. All the patients were confirmed to have COVID-19 according to the Guidelines on Diagnosis and Treatment of Novel Coronavirus Pneumonia (Trial version 6) issued by the National Health Commission and
Administration of Traditional Chinese Medicine, as well as the diagnosis guidelines issued by the WHO [11, 12]. Only patients with a positive SARS-CoV-2 reverse transcriptionpolymerase chain reaction (RT-PCR) test result from throat swab or a positive test for serum SARS-CoV-2-specific IgM were included in this study. Verbal informed consent was obtained from each patient before inclusion. Ethics approval for this study was obtained from the hospital ethics committee.

\section{Data collection}

Demographic information, medical history, basic symptoms, neurological symptoms and signs, and psychiatric presentations were recorded. Laboratory tests and chest computerized tomography (CT) scans were obtained from the electronic medical record system. The date of disease onset was defined as the day when initial symptoms appeared. The Generalized Anxiety Disorder 7-item scale (GAD-7) and Patient Health Questionnaire-9 (PHQ-9) were used to evaluate the severity of anxiety and depressed mood in patients [13-16], and the Brief Psychiatric Rating Scale (BPRS) was used to evaluate psychiatric symptoms [17-19]. Neurological symptoms were classified into three aspects: central nervous system disease and symptoms, peripheral nervous system symptoms, and muscle symptoms. All patients underwent chest CT, and cranial CT scans were performed if acute cerebral vascular disease or encephalitis was considered. All data were reviewed by a team of doctors, including well-trained neurologists and psychologists. Investigators who assisted in administering the GAD-7 and PHQ-9 questionnaires were systematically trained in advance, and unified verbal instructions were used. The meaning of each question and the correct procedure of completing the surveys were well explained, thereby allowing patients to voluntarily and independently complete the questionnaires honestly during the last 2 weeks.

The GAD-7 and PHQ-9, which are self-rating questionnaires that are specifically linked to the DSM-IV criteria of GAD and major depression, score each of the DSM-IV criteria using a scale of " $0-3$ ", with good reliability and validity (PHQ-9 has a sensitivity of $88 \%$ and a specificity of $88 \%$ for major depression and GAD-7 has a sensitivity of $89 \%$ and a specificity of $82 \%$ for GAD when their scores are greater than 10). A score of 10 or greater on the scale represents a cut point for identifying cases of GAD or major depression [13, 15]. These two instruments evaluate patients' subjective feelings in terms of anxiety and depression, respectively. The evaluation criteria for the interpretation of GAD-7 results are as follows: $0-4$, none or minimal anxiety; $5-9$, mild anxiety; $10-13$, moderate anxiety; and 14-21, severe anxiety. The evaluation criteria for the interpretation of PHQ-9 results are as follows: $0-4$, none or minimal depression; 5-9, mild depression; 
10-14, moderate depression; and 15-27, severe depression. BPRS consisting of 18 items assesses the following symptoms: somatic concern, anxiety, emotional withdrawal, conceptual disorganization, guilt feelings, tension, mannerisms and posturing, grandiosity, depressive mood, hostility, suspiciousness, hallucinatory behavior, motor retardation, uncooperativeness, unusual thought content, blunted affect, excitement, and disorientation; all items are rated on a 7-point scale (1-7) [17]. The BPRS was administered with a brief, semi-structured interview with patients, and a rather comprehensive description of major psychiatric characteristics could be obtained. Those patients whose item score was above 2 underwent a clinical assessment by a psychologist.

Peripheral blood lymphocyte subsets and pro-inflammatory factors were included in the laboratory tests. Patients with muscle soreness and elevated serum creatine kinase above the 99th percentile upper reference limit (200 U/L) [20] were defined as having muscle injuries. All neurological and psychiatric presentations were reviewed and confirmed by two well-trained neurologists and one psychologist. The severity of COVID-19 was defined according to the Guidelines on Diagnosis and Treatment of Novel Coronavirus Pneumonia (Trial version 6) and Diagnosis and Treatment of Adults with Community-acquired Pneumonia [11,21]. All patients underwent CT with a GE spiral CT scanner. Flow cytometry was used to analyze lymphocyte subsets. Throat swab specimens were collected and placed into a test tube with virus preservation fluid. RT-PCRs were subsequently performed to identify SARS-CoV-2 using detection kits approved by the China Food and Drug Administration.

\section{Statistical analysis}

IBM SPSS Statistics version 25 (IBM Corp, Armonk, NY) was used for all statistical analyses. Kolmogorov-Smirnov analyses were used to test the distribution type of continuous variables. Continuous variables with normal distributions expressed as mean and standard deviation $(x \pm \mathrm{SD})$ were compared using an independent sample $t$ test or one-way ANOVA. Continuous variables with non-normal distributions expressed as median [interquartile range (IQR)] were compared using the Mann-Whitney $U$ test. Categorical variables expressed as number (\%) were compared using the $\chi^{2}$ test or Fisher's exact test. Spearman's rank analysis was used for correlation analysis between variables. Statistical significance was defined as a $P$ value of less than 0.05 .

\section{Results}

\section{Demographic and clinical characteristics}

In total, 77 adult patients with COVID-19 were included in the study. All patients underwent a chest CT scan and blood biochemical examinations. In addition, neurological signs were examined, and psychiatric symptoms were evaluated in a face-to-face interview. A total of 65 valid GAD-7 and PHQ-9 questionnaires were collected, and 12 invalid self-rating questionnaires were excluded due to incomplete responses. Furthermore, 51 test results of peripheral blood inflammatory factors and chemokines and 54 analysis records of completed lymphocyte subsets were obtained. A number of analysis results of inflammatory cytokines and lymphocyte subsets were excluded due to incomplete data in the electronic medical record system.

Demographics and clinical characteristics are shown in Table 1. The median age was 56.0 years (IQR 43.5-63.0); 38 patients $(49.4 \%)$ were men and $39(50.6 \%)$ were women. Comorbidities in these patients included hypertension $(n=17,22.1 \%)$, diabetes mellitus $(n=5,6.5 \%)$, cardiac disease $(n=5,6.5 \%)$, cerebral vascular disease $(n=3$, $3.9 \%$ ), and malignant tumor (postoperative bladder cancer, $n=1,1.3 \%$ ). The most common symptoms during disease onset were fever $(n=75,97.4 \%)$, cough $(n=68,88.3 \%)$, fatigue $(n=28,36.4 \%)$, and diarrhea $(n=11,14.3 \%)$. In total, 18 (23.4\%) patients had neurological presentations, including headache $(n=4,5.2 \%)$, dizziness $(n=9$, $11.7 \%)$, nausea and vomiting $(n=11,14.3 \%)$, muscle soreness $(n=18,23.4 \%)$, muscle soreness combined with elevated creatine kinase $(n=4,5.2 \%)$, trigeminal neuralgia $(n=2,2.6 \%)$, tremor $(n=2,2.6 \%)$, numbness $(n=1$, $1.3 \%)$, restless leg syndrome $(n=1,1.3 \%)$, and hyposmia $(n=1,1.3 \%)$. In addition, $39(50.6 \%)$ patients had psychiatric presentations, included hallucinations $(n=1,1.3 \%)$, suicidal ideation $(n=2,2.6 \%)$, paranoia $(n=2,2.6 \%)$, agitation and aggression $(n=1,1.3 \%)$, insomnia $(n=45$, $58.4 \%)$, anxiety $(n=34 / 65,52.3 \%)$, and depressed mood $(n=31 / 65,47.7 \%)$.

According to the COVID-19 diagnostic criteria, patients were classified into either the severe $(n=23$, $29.9 \%)$ or non-severe $(n=54,70.1 \%)$ group. Severe patients received oxygen therapy through a nasal cannula. One of the severe patients was transferred to another ward for hemodialysis because of chronic renal failure. Severe patients were older [mean age 62 (range 60.0-69.0) years vs. 50 (range 38.8-60.0) years] and had more comorbidities $(82.6 \%$ vs. $22.2 \%, P<0.001)$ than non-severe patients. Among these comorbidities, chronic obstructive pulmonary disease, hypertension, and cardiac disease were more common in severe patients than in non-severe 
Table 1 Demographic and baseline characteristics of patients with COVID-19

\begin{tabular}{|c|c|c|c|c|}
\hline & $\begin{array}{l}\text { Total } \\
n=77\end{array}$ & $\begin{array}{l}\text { Non-severe } \\
n=54(70.1 \%)\end{array}$ & $\begin{array}{l}\text { Severe } \\
n=23(29.9 \%)\end{array}$ & $P$ value \\
\hline Age (years) & $56(43.5,63)$ & $50(38.8,60.0)$ & $62(60.0,69.0)$ & $<0.001$ \\
\hline \multicolumn{5}{|l|}{ Sex } \\
\hline Male & $38(49.4 \%)$ & $26(41.8 \%)$ & $12(52.2 \%)$ & \multirow[t]{2}{*}{0.746} \\
\hline Female & $39(50.6 \%)$ & $28(51.9 \%)$ & $11(47.8 \%)$ & \\
\hline Comorbidities & $31(40.3 \%)$ & $12(22.2 \%)$ & $19(82.6 \%)$ & $<0.001$ \\
\hline Chronic obstructive pulmonary disease & $3(3.9 \%)$ & $0(0.0 \%)$ & $3(13 \%)$ & 0.024 \\
\hline Hypertension & $17(22.1 \%)$ & $7(13 \%)$ & $10(43.5 \%)$ & 0.003 \\
\hline Diabetes mellitus & $5(6.5 \%)$ & $2(3.7 \%)$ & $3(13 \%)$ & 0.148 \\
\hline Heart disease & $5(6.5 \%)$ & $1(1.9 \%)$ & $4(17.4 \%)$ & 0.016 \\
\hline Kidney disease & $2(2.6 \%)$ & $0(0.0 \%)$ & $2(8.7 \%)$ & 0.086 \\
\hline Tumor & $1(1.3 \%)$ & $1(1.9 \%)$ & $0(0.0 \%)$ & 0.701 \\
\hline Cerebrovascular disease & $3(3.9 \%)$ & $1(1.9 \%)$ & $2(8.7 \%)$ & 0.211 \\
\hline \multicolumn{5}{|l|}{ Signs and symptoms } \\
\hline Fever & $75(97.4 \%)$ & $52(96.3 \%)$ & $23(100 \%)$ & 0.489 \\
\hline Cough & $68(88.3 \%)$ & $47(87.0 \%)$ & $21(91.3 \%)$ & 0.584 \\
\hline Lower extremity vein thrombosis & $1(1.3 \%)$ & $0(0.0 \%)$ & $1(4.3 \%)$ & 0.299 \\
\hline Headache & $4(5.2 \%)$ & $1(1.9 \%)$ & $3(13 \%)$ & 0.055 \\
\hline Dizziness & $9(11.7 \%)$ & $5(9.3 \%)$ & $4(17.4 \%)$ & 0.324 \\
\hline Nausea and vomiting & $11(14.3 \%)$ & $7(13.0 \%)$ & $4(17.4 \%)$ & 0.617 \\
\hline Myalgia & $18(23.4 \%)$ & $10(18.5 \%)$ & $8(34.8 \%)$ & 0.147 \\
\hline $\mathrm{CK}>200 \mathrm{U} / \mathrm{L}$ & $4(5.2 \%)$ & $3(5.6 \%)$ & $1(4.3 \%)$ & 0.824 \\
\hline Trigeminal neuralgia & $2(2.6 \%)$ & $1(1.9 \%)$ & $1(4.3 \%)$ & 0.511 \\
\hline Tremor & $2(2.6 \%)$ & $0(0.0 \%)$ & $2(8.7 \%)$ & 0.086 \\
\hline Numbness & $1(1.3 \%)$ & $0(0.0 \%)$ & $1(4.3 \%)$ & 0.299 \\
\hline Restless leg syndrome & $1(1.3 \%)$ & $1(1.9 \%)$ & $0(0.0 \%)$ & 0.701 \\
\hline Hyposmia & $1(1.3 \%)$ & $0(0.0 \%)$ & $1(4.3 \%)$ & 0.299 \\
\hline Hallucinations & $1(1.3 \%)$ & $0(0.0 \%)$ & $1(4.3 \%)$ & 0.299 \\
\hline Suicidal ideation & $2(2.6 \%)$ & $1(1.9 \%)$ & $1(4.3 \%)$ & 0.511 \\
\hline Paranoia & $2(2.6 \%)$ & $1(1.9 \%)$ & $1(4.3 \%)$ & 0.498 \\
\hline Agitation and aggression & $1(1.3 \%)$ & $1(1.9 \%)$ & $0(0.0 \%)$ & 0.701 \\
\hline Insomnia & $45(58.4 \%)$ & $28(51.9 \%)$ & $17(73.9 \%)$ & 0.072 \\
\hline Anxiety and depression & $n=65$ & $n=44$ & $n=21$ & \\
\hline GAD-7 & $5.11 \pm 4.54$ & $4.59 \pm 3.97$ & $6.19 \pm 5.50$ & 0.186 \\
\hline 5-9 points & $24(36.9 \%)$ & $16(36.4 \%)$ & $8(38.1 \%)$ & 0.892 \\
\hline $10-14$ points & $7(10.8 \%)$ & $4(9.1 \%)$ & $3(14.3 \%)$ & 0.536 \\
\hline $15-27$ points & $3(4.6 \%)$ & $1(2.3 \%)$ & $2(9.5 \%)$ & 0.242 \\
\hline PHQ-9 & $5.80 \pm 4.99$ & $4.84 \pm 4.59$ & $7.81 \pm 5.30$ & 0.024 \\
\hline 5-9 points & $13(20.0 \%)$ & $9(20.5 \%)$ & $4(19.0 \%)$ & 0.894 \\
\hline $10-14$ points & $14(21.5 \%)$ & $4(9.1 \%)$ & $10(47.6 \%)$ & 0.001 \\
\hline $15-27$ points & $4(6.2 \%)$ & $3(6.8 \%)$ & $1(4.8 \%)$ & 0.742 \\
\hline Anxious state & $34(52.3 \%)$ & $21(47.7 \%)$ & $13(61.9 \%)$ & 0.283 \\
\hline Depressive state & $31(47.7 \%)$ & $16(36.4 \%)$ & $15(71.4 \%)$ & 0.008 \\
\hline
\end{tabular}

patients $(P<0.05)$. There were no differences in fever, cough, fatigue, nausea, or vomiting between these two groups. Seven $(30.4 \%)$ patients in the severe group and $11(20.4 \%)$ patients in the non-severe group presented with neurological symptoms $\left(\chi^{2}, 0.912 ; P=0.340\right)$. There were no differences in any of the presentations mentioned above between these two groups. More patients had a depressed mood in the severe group $(15 / 21,71.4 \%)$ 
than in the non-severe group $(16 / 44,36.4 \%)(P<0.01)$. In addition, there were no differences between these two groups in any of the other psychiatric presentations.

\section{Laboratory test findings of severe and non-severe patients}

Table 2 shows the laboratory test results of severe and non-severe patients. Severe patients had significantly higher high-sensitivity C-reactive protein [hs-CRP 2.2

Table 2 Laboratory test results of patients with COVID-19

\begin{tabular}{|c|c|c|c|c|}
\hline & $\begin{array}{l}\text { Total } \\
n=51\end{array}$ & $\begin{array}{l}\text { Non-severe } \\
n=37\end{array}$ & $\begin{array}{l}\text { Severe } \\
n=14\end{array}$ & $P$ value \\
\hline IL-2 & $2.4(2.3-2.6)$ & $2.5(2.3-2.6)$ & $2.3(2.3-2.6)$ & 0.286 \\
\hline IL-4 & $2.0(1.6-2.4)$ & $2.1(1.7-2.3)$ & $2.0(1.6-2.5)$ & 0.874 \\
\hline IL-6 & $4.1(2.8-5.8)$ & $3.6(2.6-5.6)$ & $5.0(3.7-9.7)$ & 0.268 \\
\hline IL-10 & $2.8(2.3-3.2)$ & $2.8(2.3-3.2)$ & $2.7(2.4-3.3)$ & 0.908 \\
\hline TNF- $\alpha$ & $1.9(1.7-2.2)$ & $2.0(1.8-2.3)$ & $1.9(1.7-2.3)$ & 0.466 \\
\hline IFN- $\gamma$ & $1.9(1.6-2.4)$ & $1.9(1.6-2.4)$ & $1.8(1.6-2.1)$ & 0.410 \\
\hline IL-6> 2.9 & $37(72.5 \%)$ & $26(70.3 \%)$ & $11(78.6 \%)$ & 0.547 \\
\hline \multirow[t]{2}{*}{ IL-6 $<2.9$} & $14(27.5 \%)$ & $11(29.7 \%)$ & $3(21.4 \%)$ & \\
\hline & $n=54$ & $n=38$ & $n=16$ & \\
\hline $\mathrm{CD} 3+\mathrm{T}$ & $75.3(68.3-81.7)$ & $76.0(67.8-81.4)$ & $75.9(69.8-84.5)$ & 0.776 \\
\hline $\mathrm{CD} 4+\mathrm{T}$ & $46.4(40.9-52.3)$ & $46.4(42.0-51.7)$ & $48.6(38.9-52.7)$ & 0.691 \\
\hline $\mathrm{CD} 8+\mathrm{T}$ & $24.9(17.7-27.8)$ & $24.3(16.8-27.5)$ & $25.1(21.2-30.2)$ & 0.970 \\
\hline \multirow[t]{2}{*}{$\mathrm{CD} 4+/ \mathrm{CD} 8+$} & $2.1(1.5-2.6)$ & $2.1(1.5-2.9)$ & $1.9(1.5-2.3)$ & 0.483 \\
\hline & $n=77$ & $n=54$ & $n=23$ & \\
\hline Leukocyte count & $4.8(3.8-5.8)$ & $4.6(3.6-6.0)$ & $5.1(4.2-5.7)$ & 0.581 \\
\hline Neutrophil count $\left(10^{9} / \mathrm{L}\right)$ & $3.1(2.1-3.6)$ & $2.5(2.0-3.6)$ & $3.1(2.4-3.8)$ & 0.183 \\
\hline Lymphocyte count $\left(10^{9} / \mathrm{L}\right)$ & $1.4(1.1-1.8)$ & $1.5(1.2-1.9)$ & $1.3(0.9-1.6)$ & 0.143 \\
\hline Count $<1.0$ & $14(18.2 \%)$ & $7(13.0 \%)$ & $7(30.4 \%)$ & 0.078 \\
\hline Count $\geq 1.0$ & $63(81.8 \%)$ & $47(87.0 \%)$ & $16(69.6 \%)$ & \\
\hline Hemoglobin & $127.5(117.0-138.0)$ & $130.0(121.0-139.0)$ & $117.0(109.0-130.5)$ & 0.008 \\
\hline Platelet count & $193.5(167.8-247.5)$ & $193.0(167.5-244.0)$ & $215.0(165.5-274.5)$ & 0.457 \\
\hline Total bilirubin & $12.8(0.5-14.9)$ & $11.9(10.2-14.8)$ & $13.1(11.7-16.1)$ & 0.201 \\
\hline Direct bilirubin & $3.2(2.8-3.8)$ & $3.2(2.8-3.8)$ & $3.2(2.8-3.5)$ & 0.928 \\
\hline Total protein & $64.2(61.9-66.6)$ & $64.2(61.9-66.4)$ & $63.6(60.8-67.1)$ & 0.839 \\
\hline Albumin & $38.8(36.0-41.6)$ & $39.7(36.8-42.7)$ & $36.5(35.1-40.8)$ & 0.054 \\
\hline Globulin & $24.8(22.6-27.1)$ & $24.2(22.5-26.8)$ & $26.2(23.4-29.1)$ & 0.059 \\
\hline Alanine aminotransferase & $23.0(15.3-43.8)$ & $25.0(17.5-44.5)$ & $17.0(13.0-36.0)$ & 0.114 \\
\hline Aspartate transaminase & $25.5(19.3-34.5)$ & $26.0(20.0-33.0)$ & $25.0(18.0-35.0)$ & 0.618 \\
\hline Lactate dehydrogenase & $166.0(138.0-195.5)$ & $165.0(134.0-196.5)$ & $168.0(140.0-194.0)$ & 0.615 \\
\hline Creatine kinase & $71.0(50.5-98.8)$ & $71.0(57.5-88.5)$ & $61.0(42.0-131.0)$ & 0.501 \\
\hline Urea nitrogen & $4.1(3.2-4.9)$ & $3.8(3.1-4.7)$ & $4.3(3.5-7.2)$ & 0.022 \\
\hline Creatinine & $72.5(62.8-82.8)$ & $72.0(59.0-81.0)$ & $75.0(69.5-88.0)$ & 0.078 \\
\hline Cystatin C & $1.0(0.9-1.21)$ & $0.9(0.8-1.2)$ & $1.1(1.0-1.4)$ & 0.001 \\
\hline Fasting blood glucose & $5.3(5.0-5.8)$ & $5.3(4.9-5.8)$ & $5.6(5.2-5.9)$ & 0.143 \\
\hline Total cholesterol & $4.2(3.5-4.7)$ & $4.1(3.3-4.8)$ & $4.4((3.9-4.6)$ & 0.459 \\
\hline Low-density lipoprotein & $2.2(1.8-2.6)$ & $2.2(1.6-2.6)$ & $2.3(2.0-2.5)$ & 0.406 \\
\hline D-Dimer & $0.4(0.2-0.7)$ & $0.3(0.2-0.6)$ & $0.6(0.2-1.5)$ & 0.060 \\
\hline Fibrinogen & $3.7(3.2-4.7)$ & $3.6(3.1-4.3)$ & $4.4(3.5-5.9)$ & 0.020 \\
\hline Erythrocyte sedimentation rate & $30.5(18.8-62.5)$ & $22.0(17.0-59.5)$ & $35.0(24.0-75.5)$ & 0.164 \\
\hline hs-CRP & $1.6(0.5-5.3)$ & $1.3(0.3-4.8)$ & $2.2(0.9-20.4)$ & 0.039 \\
\hline
\end{tabular}


(0.9-20.4) vs. $1.3(0.3-4.8)]$, fibrinogen [4.4 (3.5-5.9) vs. $3.6(3.1-4.3)]$, urea nitrogen $[4.3(3.5-7.2)$ vs. 3.8 (3.1-4.7)], and cystatin C [1.1 (1.0-1.4) vs. $0.9(0.8-1.2)]$ levels but lower hemoglobin levels [117.0 (109.0-130.5) vs. 130.0 (121.0-139.0)] than non-severe patients $(P<0.05)$. Both groups had decreased leukocyte, neutrophil, and lymphocyte counts and an increased erythrocyte sedimentation rate (ESR), but the differences were not statistically significant. In addition serum concentrations of IL-2, IL-4, IL-6, and IL-10 were, respectively, higher than normal values by $5.9 \%$ (3/51), $7.8 \%$ (4/51), $72.5 \%(37 / 51)$, and $7.8 \%(4 / 51)$, and $\mathrm{CD} 3+, \mathrm{CD} 4+$, and $\mathrm{CD} 8+\mathrm{T}$ cell counts and the CD $4+/ \mathrm{CD} 8+\mathrm{T}$ cell ratio were, respectively, lower than normal values by $3.7 \%$ (2/54), 5.6\% (3/54), 9.3\% (5/54), and 1.9\% (1/54); however, these differences were not significant. Moreover, there were no differences in the levels of D-dimer, creatine kinase, or alanine aminotransferase between the two groups.

\section{Laboratory test findings of patients with or without neurological presentations}

Table 3 shows the laboratory test results of patients with and without neurological presentations. There were no differences in leukocyte, lymphocyte, and platelet counts, hemoglobin and D-dimer levels or ESR between the two groups. Patients with neurological presentations had lower basophil counts $(0.01 \pm 0.013$ vs. $0.02 \pm 0.007)$ but higher urea nitrogen [4.4 (3.8-6.6) vs. 3.8 (3.0-4.7)], cystatin C [1.1 $(1.0-1.4)$ vs. $1.0(0.8-1.2)]$, and hs-CRP [2.2 (0.9-20.4) vs. $1.3(0.3-4.8)$ levels $(P<0.01)$. There were no differences in IL-2, IL-4, IL-6, IL-10, TNF- $\alpha$, interferon (IFN)- $\gamma$, or lymphocyte subsets between patients with and without neurological presentations. Patients with muscle injuries had higher urea nitrogen [4.3 (3.6-5.2) vs. $3.7(3.0-4.7), P=0.045]$ and cystatin $C$ levels $[1.1(1.0-1.4)$ vs. $1.0(0.8-1.1)$, $P=0.028]$ and lower basophil counts [0.01 (0.01-0.02) vs. $0.01(0.01-0.03) ; P=0.017]$. There were no differences in neutrophil and lymphocyte counts or hs-CRP and D-dimer levels between patients with and without muscle injuries.

Table 3 Laboratory findings of patients with COVID-19 with or without neurological presentations

\begin{tabular}{|c|c|c|c|c|}
\hline & $\begin{array}{l}\text { Total } \\
n=77\end{array}$ & $\begin{array}{l}\text { Without neurological symptoms } \\
n=59\end{array}$ & $\begin{array}{l}\text { With neurological symptoms } \\
n=18\end{array}$ & $P$ value \\
\hline Leukocyte count & $4.8(3.8-5.8)$ & $5.0(4.1-6.0)$ & $4.5(3.6-5.7)$ & 0.290 \\
\hline Neutrophil count $10^{9} / \mathrm{L}$ & $3.1(2.1-3.6)$ & $3.2(2.1-3.9)$ & $2.5(1.9-3.2)$ & 0.153 \\
\hline Lymphocyte count $10^{9} / \mathrm{L}$ & $1.4(1.1-1.8)$ & $1.4(1.1-1.7)$ & $1.5(1.0-1.9)$ & 0.865 \\
\hline Basophil count & $0.01(0.01-0.02)$ & $0.01(0.01-0.03)$ & $0.01(0.01-0.02)$ & 0.017 \\
\hline Hemoglobin & $127.5(117.0-138.0)$ & $130.0(121.0-139.0)$ & $117.0(109.0-130.5)$ & 0.762 \\
\hline Platelet count & $193.5(167.8-247.5)$ & $189.5(164.8-245.0)$ & $213.0(178.0-288.2)$ & 0.254 \\
\hline Urea nitrogen & $4.1(3.2-4.9)$ & $3.8(3.0-4.7)$ & $4.4(3.8-6.6)$ & 0.045 \\
\hline Cystatin C & $1.0(0.9-1.2)$ & $1.0(0.8-1.2)$ & $1.1(1.0-1.4)$ & 0.028 \\
\hline D-Dimer & $0.4(0.2-0.7)$ & $0.3(0.2-0.7)$ & $0.5(0.2-0.7)$ & 0.647 \\
\hline Fibrinogen & $3.7(3.2-4.7)$ & $3.7(3.1-4.7)$ & $4.0(3.3-4.6)$ & 0.652 \\
\hline Erythrocyte sedimentation rate & $30.5(18.8-62.5)$ & $22.0(17.0-59.5)$ & $35.0(24.0-75.5)$ & 0.164 \\
\hline \multirow[t]{2}{*}{ hs-CRP } & $1.6(0.5-5.3)$ & $1.3(0.3-4.8)$ & $2.2(0.9-20.4)$ & 0.039 \\
\hline & $n=51$ & $n=36$ & $n=15$ & \\
\hline IL-2 & $2.4(2.3-2.6)$ & $2.4(2.3-2.6)$ & $2.4(2.3-2.6)$ & 0.456 \\
\hline IL-4 & $2.0(1.6-2.4)$ & $2.0(1.7-2.2)$ & $2.1(1.4-2.5)$ & 0.702 \\
\hline IL-6 & $4.1(2.8-5.8)$ & $4.0(2.8-5.7)$ & $4.2(2.9-19.0)$ & 0.642 \\
\hline IL-10 & $2.8(2.3-3.2)$ & $2.8(2.3-3.2)$ & $2.8(2.3-3.5)$ & 0.733 \\
\hline TNF- $\alpha$ & $1.9(1.7-2.2)$ & $1.9(1.7-2.3)$ & $1.9(1.8-2.3)$ & 0.836 \\
\hline \multirow[t]{2}{*}{ IFN- $\gamma$} & $1.9(1.6-2.4)$ & $1.8(1.6-2.5)$ & $1.9(1.6-2.0)$ & 0.627 \\
\hline & $n=54$ & $n=40$ & $n=14$ & \\
\hline $\mathrm{CD} 3+\mathrm{T}$ & $75.3(68.3-81.7)$ & $77.4(70.0-81.9)$ & $71.2(64.8-84.0)$ & 0.385 \\
\hline $\mathrm{CD} 4+\mathrm{T}$ & $46.4(40.9-52.3)$ & $46.7(42.6-52.0)$ & $47.0(36.2-55.2)$ & 0.921 \\
\hline $\mathrm{CD} 8+\mathrm{T}$ & $24.9(17.7-27.8)$ & $25.5(17.4-27.8)$ & $24.0(17.1-27.7)$ & 0.567 \\
\hline $\mathrm{CD} 4+/ \mathrm{CD} 8+$ & $2.1(1.5-2.6)$ & $2.1(1.5-2.6)$ & $2.1(1.5-2.8)$ & 0.805 \\
\hline
\end{tabular}




\section{Laboratory test findings of patients with or without psychiatric presentations}

Table 4 shows the age and laboratory test results of patients with and without psychiatric presentations, and Table 5 shows the findings of inflammatory cytokines and lymphocyte subsets in patients with anxiety and depression. Patients with psychiatric presentations were much older [60.5 (49.0-65.3) vs. $51.0(37.5-60.0) ; P<0.01]$ and had much higher IL-6 [4.9 (3.8-7.8) vs. $2.9(2.3-5.2) ; P<0.01]$ and IL-10 levels [3.0 (2.7-3.5) vs. $2.5(2.2-2.8) ; P<0.01]$ than those without psychiatric presentations. Alkaline phosphatase [71.5 (55.0-83.5) vs. 58.0 (49.0-65.5)], R-glutamate transferase [29.5 (16.8-53.3) vs. $19.0(13.0-36.0)]$, and urea nitrogen [4.4 (3.5-6.1) vs. $3.6(3.0-4.3)]$ levels were higher in patients with psychiatric presentations than in those without $(P<0.05)$. In addition, patients with anxiety had higher IL-6 [4.8 (3.8-9.2) vs. $3.4(2.4-5.8)]$ and IL-10 [3.0 (2.7-3.7) vs. $2.6(2.2-2.8)]$ levels than those without anxiety $(P<0.05)$. Furthermore, the levels of IL-6 and IL-10 were related to anxiety $(P<0.05)$. There were no differences in $\mathrm{CD} 8+$ cell counts or the $\mathrm{CD} 4+/ \mathrm{CD} 8+$ ratio between patients with and without anxiety. The levels of IL-6 and IL-10 did not differ between patients with and without depression either. However, patients with moderate depression had higher CD8 + counts [27.6 (24.4-32.2) vs. $21.9(16.1-27.5)]$ and lower CD4 +/CD8 + ratios [1.6 $(1.2-1.9)$ vs. $2.2(1.7-2.9)]$ than patients with non-moderate depression $(P<0.05)$. The changes in $\mathrm{CD} 8+$ cell counts and $\mathrm{CD} 4+/ \mathrm{CD} 8+$ ratios were significantly related to moderate depression $(P<0.05)$.

\section{Discussion}

We investigated neurological and psychiatric symptoms and their associated immune system disorders in patients with laboratory-confirmed COVID-19 in Wuhan. We found that

Table 4 Age and laboratory findings of patients with COVID-19 with or without psychiatric presentations

\begin{tabular}{|c|c|c|c|c|}
\hline & $\begin{array}{l}\text { Total } \\
n=77\end{array}$ & $\begin{array}{l}\text { Without psychiatric symptoms } \\
n=38\end{array}$ & $\begin{array}{l}\text { With psychiatric symptoms } \\
n=39\end{array}$ & $P$ value \\
\hline Age (years) & $56.0(43.5-63)$ & $51.0(37.5-60.0)$ & $60.5(49.0-65.3)$ & 0.007 \\
\hline Leukocyte count & $4.8(3.8-5.8)$ & $4.4(4.0-6.3)$ & $5.1(3.9-6.2)$ & 0.189 \\
\hline Neutrophil count $\left(10^{9} / \mathrm{L}\right)$ & $3.1(2.1-3.6)$ & $2.4(2.0-4.6)$ & $3.5(2.9-4.1)$ & 0.150 \\
\hline Lymphocyte count $\left(10^{9} / \mathrm{L}\right)$ & $1.4(1.1-1.8)$ & $1.3(1.1-1.4)$ & $1.5(1.0-1.9)$ & 0.423 \\
\hline Basophil count & $0.01(0.01-0.02)$ & $0.01(0.01-0.03)$ & $0.03(0.01-0.04)$ & 0.143 \\
\hline Hemoglobin & $127.5(117.0-138.0)$ & $130.0(121.0-139.0)$ & $117.0(109.0-130.5)$ & 0.408 \\
\hline Platelet count & $193.5(167.8-247.5)$ & $197.0(178.3-342.3)$ & $186.5(156.0-258.8)$ & 0.137 \\
\hline Alkaline phosphatase & $61.0(52.0-78.8)$ & $58.0(49.0-65.5)$ & $71.5(55.0-83.5)$ & 0.006 \\
\hline R-glutamyl transferase & $26.5(15.0-40.8)$ & $19.0(13.0-36.0)$ & $29.5(16.8-53.3)$ & 0.026 \\
\hline Urea nitrogen & $4.1(3.2-4.9)$ & $3.6(3.0-4.3)$ & $4.4(3.5-6.1)$ & 0.014 \\
\hline Cystatin C & $1.0(0.9-1.2)$ & $1.0(0.8-1.1)$ & $1.1(1.0-1.2)$ & 0.115 \\
\hline D-Dimer & $0.4(0.2-0.7)$ & $0.6(0.2-0.7)$ & $0.4(0.2-0.5)$ & 0.213 \\
\hline Fibrinogen & $3.7(3.2-4.7)$ & $4.0(3.2-5.1)$ & $4.5(4.1-5.7)$ & 0.582 \\
\hline Erythrocyte sedimentation rate & $30.5(18.8-62.5)$ & $19.5(16.5-55.5)$ & $40.0(21.2-77.8)$ & 0.382 \\
\hline \multirow[t]{2}{*}{ hs-CRP } & $1.6(0.5-5.3)$ & $1.1(0.3-5.5)$ & $4.5(1.4-14.2)$ & 0.234 \\
\hline & $n=51$ & $n=36$ & $n=15$ & \\
\hline IL-2 & $2.4(2.3-2.6)$ & $2.4(2.3-2.5)$ & $2.5(2.3-2.7)$ & 0.317 \\
\hline IL-4 & $2.0(1.6-2.4)$ & $1.8(1.6-2.3)$ & $2.1(1.7-2.4)$ & 0.270 \\
\hline IL-6 & $4.1(2.8-5.8)$ & $2.9(2.3-5.2)$ & $4.9(3.8-7.8)$ & 0.002 \\
\hline IL-10 & $2.8(2.3-3.2)$ & $2.5(2.2-2.8)$ & $3.0(2.7-3.5)$ & 0.004 \\
\hline TNF- $\alpha$ & $1.9(1.7-2.2)$ & $1.9(1.7-2.2)$ & $2.0(1.7-2.4)$ & 0.231 \\
\hline \multirow[t]{2}{*}{ IFN- $\gamma$} & $1.9(1.6-2.4)$ & $1.8(1.5-2.2)$ & $1.9(1.7-2.6)$ & 0.172 \\
\hline & $n=54$ & $n=40$ & $n=14$ & \\
\hline $\mathrm{CD} 3+\mathrm{T}$ & $75.3(68.3-81.7)$ & $78.0(69.3-82.1)$ & $73.6(65.8-81.6)$ & 0.332 \\
\hline $\mathrm{CD} 4+\mathrm{T}$ & $46.4(40.9-52.3)$ & $45.5(41.6-52.1)$ & $47.4(42.5-52.3)$ & 0.822 \\
\hline $\mathrm{CD} 8+\mathrm{T}$ & $24.9(17.7-27.8)$ & $25.4(19.3-28.9)$ & $24.1(16.7-27.8)$ & 0.604 \\
\hline $\mathrm{CD} 4+/ \mathrm{CD} 8+$ & $2.1(1.5-2.6)$ & $2.0(1.4-2.7)$ & $2.1(1.6-2.6)$ & 0.952 \\
\hline
\end{tabular}


Table 5 Findings of inflammatory cytokines and lymphocyte subsets in patients with COVID-19 with anxiety and depression

\begin{tabular}{|c|c|c|c|c|c|c|c|c|c|c|c|}
\hline & & IL-2 & IL-4 & IL-6 & IL-10 & TNF- $\alpha$ & IFN-r & $\mathrm{CD} 3+$ & $\mathrm{CD} 4+$ & $\mathrm{CD} 8+$ & $\overline{\mathrm{CD} 4+/ \mathrm{CD} 8+}$ \\
\hline \multicolumn{12}{|c|}{ Total patients with anxiety } \\
\hline \multirow[t]{2}{*}{ Spearman } & $r$ & 0.011 & 0.173 & 0.301 & 0.430 & 0.075 & 0.228 & -0.068 & 0.107 & -0.001 & -0.008 \\
\hline & $P$ & 0.942 & 0.246 & 0.040 & 0.003 & 0.615 & 0.124 & 0.639 & 0.461 & 0.992 & 0.954 \\
\hline \multirow[t]{2}{*}{ Mann-Whitney } & $U$ & 272.5 & 221.0 & 180.0 & 139.0 & 252.0 & 203.5 & 288.0 & 274.0 & 312.0 & 309.5 \\
\hline & $P$ & 0.941 & 0.242 & 0.041 & 0.004 & 0.609 & 0.123 & 0.635 & 0.455 & 0.992 & 0.954 \\
\hline \multicolumn{12}{|c|}{ Patients with moderate anxiety } \\
\hline \multirow[t]{2}{*}{ Spearman } & $r$ & 0.127 & -0.008 & 0.287 & 0.321 & 0.048 & 0.226 & -0.200 & -0.043 & -0.009 & -0.060 \\
\hline & $P$ & 0.394 & 0.959 & 0.050 & 0.028 & 0.747 & 0.126 & 0.163 & 0.769 & 0.953 & 0.680 \\
\hline \multirow[t]{2}{*}{ Mann-Whitney } & $U$ & 80.0 & 103.5 & 48.5 & 42.0 & 95.5 & 60.5 & 85.0 & 122.0 & 130.0 & 118.0 \\
\hline & $P$ & 0.409 & 0.960 & 0.049 & 0.028 & 0.751 & 0.128 & 0.169 & 0.782 & 0.965 & 0.694 \\
\hline \multicolumn{12}{|c|}{ Total patients with depression } \\
\hline \multirow[t]{2}{*}{ Spearman } & $r$ & 0.131 & 0.196 & 0.276 & 0.238 & 0.131 & 0.196 & -0.034 & 0.034 & 0.081 & -0.113 \\
\hline & $P$ & 0.380 & 0.187 & 0.060 & 0.107 & 0.380 & 0.187 & 0.817 & 0.817 & 0.576 & 0.434 \\
\hline \multirow[t]{2}{*}{ Mann-Whitney } & $U$ & 231.5 & 211.0 & 185.5 & 197.5 & 250.5 & 208.5 & 296.0 & 296.0 & 279.0 & 267.5 \\
\hline & $P$ & 0.374 & 0.184 & 0.061 & 0.106 & 0.630 & 0.167 & 0.815 & 0.815 & 0.571 & 0.429 \\
\hline \multicolumn{12}{|c|}{ Patients with moderate depression } \\
\hline \multirow[t]{2}{*}{ Spearman } & $r$ & 0.084 & 0.274 & 0.140 & 0.230 & 0.084 & 0.274 & 0.069 & -0.121 & 0.308 & -0.352 \\
\hline & $P$ & 0.573 & 0.062 & 0.348 & 0.120 & 0.573 & 0.062 & 0.633 & 0.402 & 0.029 & 0.012 \\
\hline \multirow[t]{2}{*}{ Mann-Whitney } & $U$ & 163.0 & 113.5 & 148.5 & 125.0 & 163.0 & 150.5 & 180.0 & 165.0 & 111.0 & 98.5 \\
\hline & $P$ & 0.582 & 0.062 & 0.348 & 0.123 & 0.582 & 0.376 & 0.641 & 0.409 & 0.030 & 0.012 \\
\hline
\end{tabular}

nearly one-fifth of the patients had neurological symptoms, and half had psychiatric symptoms. Basophils were lower, whereas blood urea nitrogen, cystatin $\mathrm{C}$, and hypersensitivity C-reactive protein levels were significantly higher in patients with neurological symptoms. Patients with muscle injury had higher urea and Cystatin $\mathrm{C}$ levels and lower basophil levels. In addition, the age was significantly higher, and IL-6, IL-10, alkaline phosphatase, r-glutamate transferase, and urea nitrogen levels were significantly increased in patients with psychiatric symptoms compared with those without. Moreover, patients with anxiety had higher serum IL-6 and IL-10 levels, while patients with moderate depression had higher CD8 + levels and lower CD4+/CD8 + ratios. These findings suggest that neurological and psychiatric presentations are associated with increased expression levels of pro-inflammatory factors and serum $\mathrm{T}$ cell subsets in patients with COVID-19.

Coronavirus can affect multiple organ systems, including the gastrointestinal and respiratory tract, in different species, especially vertebrates. All $\beta$-type coronaviruses can potentially invade the nervous system of humans and animals [9, 22], and this infection spreads through hematogenous transmission or retrograde infection along neurons. This present study observed neurological presentations, including headache, nausea and vomiting, muscle soreness, muscle soreness combined with elevated creatine kinase, trigeminal neuralgia, tremor, numbness, restless leg syndrome, and hyposmia, and psychiatric presentations, including hallucinations, suicidal ideation, paranoia, agitation and aggression, insomnia, anxiety, and depressed mood in patients with confirmed COVID-19. Notably, studies have shown that SARS-CoV and SARS-CoV-2 can infect humans through ACE2 receptors. ACE2 is widely present in the respiratory tract, lungs, small intestine, cardiac muscle, kidneys, testes, oral cavity cell membranes, neurons, and skeletal muscle [22-26], and the extent of the severity of SARS-CoV-2 infection is related to the expression and distribution of the ACE2 receptor. Therefore, SARS-CoV-2 might infect the nervous system and cause injury through the ACE2 receptor. In addition, infection-mediated overreaction of the immune system and aberrant serum pro-inflammatory factors might also cause nervous system injuries in COVID19 patients.

Previous studies have demonstrated that patients infected with SARS-CoV have dramatic peripheral lymphopenia with decreased CD8 + and CD4 + T cell counts [4, 27, 28] and altered activation of CD4 + and $\mathrm{CD} 8+\mathrm{T}$ cells [27]. These changes are in line with the disease severity at the acute stage of the SARS infection [27]. Consistently, our study found lymphopenia; altered $\mathrm{CD} 3+, \mathrm{CD} 4+$, and $\mathrm{CD} 8+\mathrm{T}$ cell counts; and a decreased $\mathrm{CD} 4+/ \mathrm{CD} 8+$ ratio in patients infected with SARS-CoV-2. T lymphocyte subsets are a group of cells that play an important role in the immune system, and $\mathrm{CD} 3+, \mathrm{CD} 4+$, and CD8 + T cells widely participate in the immune response, acting to balance each other's responses. Patients infected with SARS-CoV-2 experience 
lymphopenia and might have an induced inflammatory cytokine storm, consequently leading to a series of immune responses, inflammation, and organ damage [29, 30]. After activation, $\mathrm{T}$ cells proliferate vigorously and migrate to the site of infection, and activated $\mathrm{T}$ cells produce inflammatory cytokines, chemokines, and cytotoxic molecules, which can inhibit virus replication and remove the virus [27]. Importantly, altered antigen-presenting cell function and impaired dendritic cell migration result in reduced priming of T cells [27, 31]. Other possible explanations for T cell lymphopenia include $\mathrm{T}$ cell apoptosis caused by increased IFNs and stress response [27].

In the SARS- and MERS-related cytokine storm, severe patients exhibit an over-production of pro-inflammatory cytokines (IFN-y, TNF- $\alpha$, IL-6, IL-12, IL-15, and IL-17) and chemokines (CXCL10 and IL-8), but a lack of IL-10 production [32]. IL-10 can inhibit the expression of many pro-inflammatory cytokines, chemokines, and chemokine receptors, thereby preventing a cytokine storm. One study showed that IL-2, IL-7, IL-10, and TNF- $\alpha$ levels were dramatically increased in severe patients infected with SARS-CoV-2 [5]. In this present study, we found remarkably increased serum IL-2, IL-4, IL-6, and IL-10 levels in severe patients, especially IL-6 levels, which were above the normal level in approximately $72.5 \%$ of the patients. In addition, $7.8 \%$ of the patients had increased IL-10 levels above the normal level. The finding (increased IL-10 levels in SARS-CoV-2 patients) is different from that in SARS and MERS patients, the underlying mechanism should be further clarified. In our study, the findings mentioned above did not differ between the severe and non-severe groups. One possible reason is that our cases were relatively mild and did not include critically ill cases.

We adopted a BPRS-based semi-structured interview combined with the self-rating scale in this study and found that $50.6 \%$ of the patients experienced psychiatric symptoms. BPRS is the most established quantitative scale for rapid clinical assessment of inpatients' mental state. We employed the original BPRS but standardized the 18-item version to improve efficiency [33]. In addition, we used GAD-7 and PHQ-9 to evaluate the anxiety and depressive symptoms of patients with COVID-19. Our survey showed that the PHQ-9 scores of severe patients were significantly higher than those of non-severe patients, suggesting that the incidence of depressive symptoms is higher in severe patients. The progression of COVID-19 may aggravate patients' depression and affect immune function. Indeed, our study showed that patients with psychiatric presentations and anxiety had higher IL-6 and IL-10 levels, and IL levels were significantly related to anxiety. However, there were no differences in IL-6 and IL-10 levels between patients with and without depression. Some evidence shows that cytokines are not only regulators of immune cell interactions but also key factors for the interaction between the immune system and the central nervous system. Cytokines play a key role in signaling to the brain to mount a neurochemical, neuroendocrine, neuroimmune, and behavioral response [34], but abnormal cytokine signaling can cause nervous system damage and cognitive behavior impairment by changing neurotransmitter biosynthesis and desensitizing the hypothalamic-pituitary-adrenal axis (HPA-axis) [35]. In fact, IL-6 has both neuroprotective and neurotoxic effects in the central nervous system: normal expression of IL-6 can enhance neurogenesis and nerve repair, but a high expression of IL-6 can lead to nerve injury and inhibition, neurotransmitter dysfunction, and oxidative stress [36]. Therefore, inflammatory cytokines, such as IL-6, may mediate the occurrence of neuropsychiatric symptoms in patients infected with SARSCoV-2. Notably, studies have shown that severe depression, generalized anxiety, post-traumatic stress disorder, and other neuropsychiatric diseases are associated with increased expression levels of inflammatory cytokines, including IL-6, IL-8, IL-10, TNF- $\alpha$, and their receptors, and higher CRP levels $[34,37,38]$. Our finding also supports the notion that cytokines play an important role in the pathophysiology of neuropsychiatric symptoms. IL-6 modulates the HPA-axis, and the stimulation of this axis would lead to increased secretion of corticotropin-releasing hormone, adrenocorticotropic hormone, and cortisol and increased turnover of serotonin and catecholamines. These changes in hormones and neurotransmitters will lead to HPA-axis disturbance. In addition, IL-6 induces production of indoleamine 2,3-dioxygenase, leading to a decrease in tryptophan and the production of tryptophan catabolites, which are associated with depressive symptoms $[39,40]$. Our study showed that patients with moderate depression had significantly higher $\mathrm{CD} 8+\mathrm{T}$ cell counts and lower $\mathrm{CD} 4+/ \mathrm{CD} 8+$ ratios than those with non-moderate depression. This finding suggests that an imbalance of immune function, especially over-activation of cellular immunity, might be a pathological basis for depression in patients with COVID-19.

Further, our study found that patients with neurological presentations had lower basophil counts but higher urea nitrogen, cystatin C, and hs-CRP levels than those without neurological presentations. After re-stimulation with a soluble antigen, basophils are the main source of IL-6 and IL- 4 in the spleen and bone marrow and key contributors to the humoral memory immune response [41]. The mechanism underlying the effect of basophils in the nervous system is still unclear. During activation, basophils produce and secrete a large number of inflammatory mediators, including histamine and leukotriene C4 [42], which could cause nervous system damage. Studies have found that cystatin $\mathrm{C}$ (a cysteine protease inhibitor) has a variety of biological effects on the brain is also an inflammatory factor, which is involved the induction of microglia 
activation [43, 44]. Increased levels of cystatin $\mathrm{C}$ might damage nerve cells in patients with COVID-19.

Notably, in our study, patients in the severe group were older than those in the non-severe group, and had more comorbidities. Severe patients had lower hemoglobin levels but higher fibrinogen, CRP, urea nitrogen, and cystatin $\mathrm{C}$ levels. These findings indicate that age and underlying diseases may be risk factors for serious complications and even death in COVID-19 patients. These results are in line with those in a previous study [4].

Our study has several limitations. First, the sample size was relatively small, and no critically ill patients were included; in addition, there was a lack of a nonCOVID hospitalized control group. These factors might lead to a bias in clinical observation. Second, the types of cytokines measured in our study were limited, and only six cytokines were measured and analyzed. Other cytokines or chemokines might also play important roles. Therefore, future studies are needed to analyze expression levels of other cytokines or chemokines in COVID-19 patients. Third, this study mainly used BPRS and self-reported questionnaires to measure psychiatric symptoms, and patients were not clinically diagnosed. The gold standard for establishing psychiatric diagnosis involves a structured clinical interview and functional neuroimaging [45, 46].

To the best of our knowledge, this study is the first to investigate both neurological and psychiatric presentations in patients with COVID-19. Our findings suggest that more attention should paid to neurological and psychiatric presentations in patients with COVID-19. During the epidemic, some patients may initially present with neuropsychiatric symptoms, and physicians need to be aware of SARS-CoV-2 infection when dealing with these patients. SARS-CoV-2 infections involve not only the respiratory system but also the nervous system and skeletal muscle. Damage to the central nervous system may present as both neurological and psychiatric symptoms. While the underlying pathophysiology is unclear, possible mechanisms include direct virus invasion of the central nervous system, infection-mediated overreaction of the immune system, and aberrant serum pro-inflammatory factors. Basophils and cystatin $\mathrm{C}$ may also play a role in the pathological processes. As for treatment, neurological and psychiatric presentations should also be evaluated and managed as needed. Future studies are needed to unveil the underlying mechanism of neurological and psychiatric impairments and to investigate the influence of neurological and psychiatric presentations on the prognosis in patients with COVID-19.

Author contribution $\mathrm{XH}$ and $\mathrm{XH}$ developed the concept and design of the study, had full access to all the data used in the study, and take responsibility for the integrity of all patient data and the accuracy of the data analysis. Drafting of the manuscript: $\mathrm{XH}$. Statistical analysis: $\mathrm{XH}$ and DZ. Critical revision of the manuscript: XH, XH, LZ, and DZ. Data acquisition: XH, LZ, XZ, LZ, GZ, KP, and HY.

Funding This research received no specific grant from any funding agency in the public, commercial, or not-for-profit sectors.

Data availability Data are available upon reasonable request. All data access requests should be directed to the corresponding author $(\mathrm{XH})$.

\section{Declarations}

Conflict of interest The authors declare that they have no conflict of interests.

Ethical approval This study was approved by the hospital ethics committee (The ethics committee of Sir Run Run Shaw hospital, College of Medicine, Zhejiang University; Approval number, 00331/2020). All protocols were in accordance with the ethical standards laid down in the 1964 Declaration of Helsinki and its later amendments.

Informed consent Informed consent was obtained from all individual participants included in the study before registration.

\section{References}

1. National Health Commission of the People's Republic of China (2020) Daily briefing on novel coronavirus cases in China. http:// www.nhc.gov.cn/xcs/yqtb/202003/0fc43d6804b04a4595a2eadd8 46c0a6e.shtml. Accessed March 192020

2. World Health Organization (2020) Coronavirus disease (COVID19) weekly epidemiological update and weekly operational update: situation reports. https://www.who.int/emergencies/disea ses/novel-coronavirus-2019/situation-reports. Accessed March 19 2020

3. Zhou P, Yang XL, Wang XG, Hu B, Zhang L, Zhang W, Si HR, Zhu Y, Li B, Huang CL, Chen HD, Chen J, Luo Y, Guo H, Jiang RD, Liu MQ, Chen Y, Shen XR, Wang X, Zheng XS, Zhao K, Chen QJ, Deng F, Liu LL, Yan B, Zhan FX, Wang YY, Xiao GF, Shi ZL (2020) A pneumonia outbreak associated with a new coronavirus of probable bat origin. Nature 579:270-273. https:// doi.org/10.1038/s41586-020-2012-7

4. World Health Organization (2020) WHO Director-General's remarks at the media briefing on 2019-nCoV. https://www.who. $\mathrm{int} / \mathrm{dg} /$ speeches/detail/who-director-general-s-remarks-at-themedia-briefing-on-2019-ncov-on-11-february-2020. Accessed February 112020

5. Coronaviridae Study Group of the International Committee on Taxonomy of Viruses (2020) The species severe acute respiratory syndrome-related coronavirus: classifying 2019-nCoV and naming it SARS-CoV-2. Nat Microbiol 5(4):536-544. https://doi.org/ 10.1038/s41564-020-0695-Z

6. Enserink M (2003) Infectious diseases. Calling all coronavirologists. Science 300:413-414. https://doi.org/10.1126/science.300. 5618.413

7. Chen N, Zhou M, Dong X, Qu J, Gong F, Han Y, Qiu Y, Wang J, Liu Y, Wei Y, Xia J, Yu T, Zhang X, Zhang L (2020) Epidemiological and clinical characteristics of 99 cases of 2019 novel coronavirus pneumonia in Wuhan, China: a descriptive study. Lancet 395:507-513. https://doi.org/10.1016/s0140-6736(20)30211-7

8. Huang C, Wang Y, Li X, Ren L, Zhao J, Hu Y, Zhang L, Fan G, Xu J, Gu X, Cheng Z, Yu T, Xia J, Wei Y, Wu W, Xie X, Yin W, 
Li H, Liu M, Xiao Y, Gao H, Guo L, Xie J, Wang G, Jiang R, Gao Z, Jin Q, Wang J, Cao B (2020) Clinical features of patients infected with 2019 novel coronavirus in Wuhan, China. Lancet 395:497-506. https://doi.org/10.1016/S0140-6736(20)30183-5

9. Glass WG, Subbarao K, Murphy B, Murphy PM (2004) Mechanisms of host defense following severe acute respiratory syndrome-coronavirus (SARS-CoV) pulmonary infection of mice. J Immunol 173:4030-4039. https://doi.org/10.4049/jimmunol. 173.6.4030

10. Hao F, Tam W, Hu X, Tan W, Jiang L, Jiang X, Zhang L, Zhao X, Zou Y, Hu Y, Luo X, McIntyre RS, Quek T, Tran BX, Zhang Z, Pham HQ, Ho CSH (2020) Ho RCM (2020) A quantitative and qualitative study on the neuropsychiatric sequelae of acutely ill COVID-19 inpatients in isolation facilities. Transl Psychiatry 10(1):355. https://doi.org/10.1038/s41398-020-01039-2

11. National Health Commission of the People's Republic of China (2020) Guidelines on diagnosis and treatment of novel coronavirus pneumonia. Chin J Infect Control 19:192-195. https://doi.org/ 10.12138/j.issn.1671-9638.20206154

12. World Health Organization (2020) Coronavirus disease (COVID19) technical guidance: patient management. https://www.who.int/ emergencies/diseases/novel-coronavirus-2019/technical-guidance/ patient-management. Accessed January 282020

13. Spitzer RL, Kroenke K, Williams JB, Löwe B (2006) A brief measure for assessing generalized anxiety disorder: the GAD-7. Arch Intern Med 166(10):1092-1097. https://doi.org/10.1001/ archinte.166.10.1092

14. Löwe B, Decker O, Müller S, Brähler E, Schellberg D, Herzog W, Herzberg PY (2008) Validation and standardization of the generalized anxiety disorder screener (GAD-7) in the general population. Med Care 46:266-274. https://doi.org/10.1097/MLR.0b013e3181 60d093

15. Kroenke K, Spitzer RL, Williams JB (2001) The PHQ-9: validity of a brief depression severity measure. J Gen Intern Med 16(9):606-613. https://doi.org/10.1046/j.1525-1497.2001.01600 9606.x

16. Levis B, Benedetti A, Thombs BD (2019) Accuracy of patient health questionnaire-9 (PHQ-9) for screening to detect major depression: individual participant data meta-analysis. BMJ 365:11476. https://doi.org/10.1136/bmj.11476

17. Overall JE, Gorham, DR (1988). The brief psychiatric rating scale (BPRS): recent developments in ascertainment and scaling. Psychopharmacology Bulletin, 24, 97-99. https://medworksmedia. com/product/brief-psychiatric-rating-scale/

18. Woerner MG, Mannuzza S, Kane JM (1988) Anchoring the BPRS: an aid to improved reliability. Psychopharmacol Bull 24(1):112-117

19. Lachar D, Bailley SE, Rhoades HM, Espadas A, Aponte M, Cowan KA, Gummattira P, Kopecky CR, Wassef A (2001) New subscales for an anchored version of the brief psychiatric rating scale: construction, reliability, and validity in acute psychiatric admissions. Psychol Assess 13(3):384-395. https://doi.org/10. 1037//1040-3590.13.3.384

20. Gao C, Wang Y, Gu X, Shen X, Zhou D, Zhou S, Huang JA, Cao B, Guo Q (2020) Association between cardiac injury and mortality in hospitalized patients infected with avian influenza A (H7N9) virus. Crit Care Med 48:451-458. https://doi.org/10.1097/ccm. 0000000000004207

21. Metlay JP, Waterer GW, Long AC, Anzueto A, Brozek J, Crothers K, Cooley LA, Dean NC, Fine MJ, Flanders SA, Griffin MR, Metersky ML, Musher DM, Restrepo MI, Whitney CG (2019) Diagnosis and treatment of adults with community-acquired pneumonia: an official clinical practice guideline of the American Thoracic Society and infectious diseases society of America. Am J Respir Crit Care Med 200:e45-e67. https://doi.org/10.1164/rccm. 201908-1581st
22. Gu J, Korteweg C (2007) Pathology and pathogenesis of severe acute respiratory syndrome. Am J Pathol 170:1136-1147. https:// doi.org/10.2353/ajpath.2007.061088

23. Guo Y, Korteweg C, McNutt MA, Gu J (2008) Pathogenetic mechanisms of severe acute respiratory syndrome. Virus Res 133:4-12. https://doi.org/10.1016/j.virusres.2007.01.022

24. Hamming I, Timens W, Bulthuis ML, Lely AT, Navis G, van Goor H (2004) Tissue distribution of ACE2 protein, the functional receptor for SARS coronavirus. A first step in understanding SARS pathogenesis. J Pathol 203:631-637. https://doi.org/10. 1002/path. 1570

25. Wang Z, Xu X (2020) scRNA-seq profiling of human testes reveals the presence of the ACE2 receptor, a target for SARSCoV-2 infection in spermatogonia. Leydig and Sertoli Cells Cells 9:920. https://doi.org/10.3390/cells9040920

26. Xu H, Zhong L, Deng J, Peng J, Dan H, Zeng X, Li T, Chen Q (2020) High expression of ACE2 receptor of 2019-nCoV on the epithelial cells of oral mucosa. Int J Oral Sci 12:8. https://doi.org/ 10.1038/s41368-020-0074-x

27. Channappanavar R, Zhao J, Perlman S (2014) T cell-mediated immune response to respiratory coronaviruses. Immunol Res 59:118-128. https://doi.org/10.1007/s12026-014-8534-Z

28. Qin C, Zhou L, Hu Z, Zhang S, Yang S, Tao Y, Xie C, Ma K, Shang K, Wang W, Tian DS (2020) Dysregulation of immune response in patients With coronavirus 2019 (COVID-19) in Wuhan. China Clin Infect Dis 71(15):762-768. https://doi.org/ 10.1093/cid/ciaa248

29. Wong CK, Lam CW, Wu AK, Ip WK, Lee NL, Chan IH, Lit LC, Hui DS, Chan MH, Chung SS, Sung JJ (2004) Plasma inflammatory cytokines and chemokines in severe acute respiratory syndrome. Clin Exp Immunol 136:95-103. https://doi.org/10.1111/j. 1365-2249.2004.02415.x

30. Xu Z, Shi L, Wang Y, Zhang J, Huang L, Zhang C, Liu S, Zhao P, Liu H, Zhu L, Tai Y, Bai C, Gao T, Song J, Xia P, Dong J, Zhao J, Wang FS (2020) Pathological findings of COVID-19 associated with acute respiratory distress syndrome. Lancet Respir Med 8:420-422. https://doi.org/10.1016/S2213-2600(20)30076-X

31. Zhao J, Zhao J, Van Rooijen N, Perlman S (2009) Evasion by stealth: inefficient immune activation underlies poor $\mathrm{T}$ cell response and severe disease in SARS-CoV-infected mice. PLoS Pathog 5:e1000636. https://doi.org/10.1371/journal.ppat.1000636

32. Chien JY, Hsueh PR, Cheng WC, Yu CJ, Yang PC (2006) Temporal changes in cytokine/chemokine profiles and pulmonary involvement in severe acute respiratory syndrome. Respirology 11:715-722. https://doi.org/10.1111/j.1440-1843.2006.00942.x

33. Hafkenscheid A (1991) Psychometric evaluation of a standardized and expanded Brief Psychiatric Rating Scale. Acta Psychiatr Scand 84(3):294-300. https://doi.org/10.1111/j.1600-0447.1991. tb03147.x

34. Hou R, Garner M, Holmes C, Osmond C, Teeling J, Lau L, Baldwin DS (2017) Peripheral inflammatory cytokines and immune balance in generalised anxiety disorder: case-controlled study. Brain Behav Immun 62:212-218. https://doi.org/10.1016/j.bbi. 2017.01.021

35. Visentin APV, Colombo R, Scotton E, Fracasso DS, da Rosa AR, Branco CS, Salvador M (2020) Targeting inflammatory-mitochondrial response in major depression: current evidence and further challenges. Oxid Med Cell Longev 2020:2972968. https://doi.org/ 10.1155/2020/2972968

36. Kim YK, Na KS, Myint AM, Leonard BE (2016) The role of pro-inflammatory cytokines in neuroinflammation, neurogenesis and the neuroendocrine system in major depression. Prog Neuropsychopharmacol Biol Psychiatry 64:277-284. https://doi.org/ 10.1016/j.pnpbp.2015.06.008

37. Uint L, Bastos GM, Thurow HS, Borges JB, Hirata TDC, França JID, Hirata MH, Sousa AGMR (2019) Increased levels of plasma 
IL-1b and BDNF can predict resistant depression patients. Rev Assoc Med Bras 65:361-369. https://doi.org/10.1590/1806-9282. 65.3.361

38. Kiecolt-Glaser JK, Derry HM, Fagundes CP (2015) Inflammation: depression fans the flames and feasts on the heat. Am J Psychiatry 172:1075-1091. https://doi.org/10.1176/appi.ajp.2015.15020152

39. Ng A, Tam WW, Zhang MW, Ho CS, Husain SF, McIntyre RS, Ho RC (2018) IL-1 $\beta$, IL-6, TNF- $\alpha$ and CRP in elderly patients with depression or Alzheimer's disease: systematic review and meta-analysis. Sci Rep 8(1):12050. https://doi.org/10.1038/ s41598-018-30487-6

40. Liu Y, Ho RC, Mak A (2012) Interleukin (IL)-6, tumour necrosis factor alpha $(\mathrm{TNF}-\alpha)$ and soluble interleukin-2 receptors (sIL-2R) are elevated in patients with major depressive disorder: a metaanalysis and meta-regression. J Affect Disord 139(3):230-239. https://doi.org/10.1016/j.jad.2011.08.003 (Epub 2011 August 26)

41. Denzel A, Maus UA, Rodriguez Gomez M, Moll C, Niedermeier M, Winter C, Maus R, Hollingshead S, Briles DE, KunzSchughart LA, Talke Y, Mack M (2008) Basophils enhance immunological memory responses. Nat Immunol 9:733-742. https://doi. org/10.1038/ni.1621

42. Pecaric-Petkovic T, Didichenko SA, Kaempfer S, Spiegl N, Dahinden CA (2009) Human basophils and eosinophils are the direct target leukocytes of the novel IL-1 family member IL-33. Blood 113:1526-1534. https://doi.org/10.1182/blood-2008-05-157818

43. Xu Y, Schnorrer P, Proietto A, Kowalski G, Febbraio MA, AchaOrbea H, Dickins RA, Villadangos JA (2011) IL-10 controls cystatin $\mathrm{C}$ synthesis and blood concentration in response to inflammation through regulation of IFN regulatory factor 8 expression. J Immunol 186:3666-3673. https://doi.org/10.4049/jimmunol. 1001934

44. Wada M, Nagasawa H, Kawanami T, Kurita K, Daimon M, Kubota I, Kayama T, Kato T (2010) Cystatin C as an index of cerebral small vessel disease: results of a cross-sectional study in community-based Japanese elderly. Eur J Neurol 17:383-390. https://doi.org/10.1111/j.1468-1331.2009.02809.x

45. Husain SF, Yu R, Tang TB, Tam WW, Tran B, Quek TT, Hwang SH, Chang CW, Ho CS (2020) Validating a functional nearinfrared spectroscopy diagnostic paradigm for major depressive disorder. Sci Rep 10(1):9740. https://doi.org/10.1038/ s41598-020-66784-2

46. Ho CSH, Lim LJH, Lim AQ, Chan NHC, Tan RS, Lee SH, Ho RCM (2020) (2020) Diagnostic and predictive applications of functional near-infrared spectroscopy for major depressive disorder: a systematic review. Front Psychiatry 11:378. https://doi.org/ $10.3389 /$ fpsyt.2020.00378 